\title{
DPPH Free-radical Scavenging and Cytotoxic Activities of Leeamacrophylla
}

\author{
Ahmed $\mathrm{F}^{1 *}$, Akter $\mathrm{D}^{2}$, Muhit $\mathrm{MA}^{3}$, Raihan $\mathrm{SZ}^{3}$, Faroque $\mathrm{ABM}^{4}$ \\ ${ }^{1}$ Department of Pharmaceutical Chemistry, University of Dhaka, Dhaka, Bangladesh \\ ${ }^{2}$ Department of Pharmacy, University of Dhaka, Dhaka, Bangladesh \\ ${ }^{3}$ Department of Clinical Pharmacy and Pharmacology, University of Dhaka, Dhaka, Bangladesh \\ ${ }^{4}$ Department of Pharmaceutical Technology, University of Dhaka, Dhaka, Bangladesh
}

\begin{abstract}
Structural damage inflicted to a wide variety of bioorganic compounds (DNA, protein, carbohydrates, and lipid) may occur as a consequence of oxidative damage which is naturally attributed to our daily lifestyle. A number of mechanisms are exploited to neutralize the reactive oxygen species. Medicinal plant can play a pivotal role of detoxifying the generated ROS. Leeamacrophylla (Leeaceae), a wild medicinal plant, has been taken into consideration of having antioxidant and cytotoxic properties. In this study, in vitro DPPH free radical scavenging activity, total phenolic content and total flavonoid content of methanol extract from the leaves of L. macrophylla were determined with using UV spectrophotometric method. Antioxidant activity of the crude extract was expressed as percentage of DPPH free radical inhibition and $\mathrm{IC}_{50}$ value $(\mu \mathrm{g} / \mathrm{ml})$. The $\mathrm{IC}_{50}$ value of $L$. macrophylla was found to be $152.40 \mu \mathrm{g} / \mathrm{ml}$. Compared to standard ascorbic acid $(4.36 \mu \mathrm{g} / \mathrm{ml})$, L. macrophylla leaf extract has moderate antioxidant property. The total phenolic content was $65.75 \mathrm{mg} / \mathrm{g}$ of dry weight of extract, expressed as gallic acid equivalents. The total flavonoid concentration was $59.8 \mathrm{mg} / \mathrm{g}$, expressed as quercetin equivalents. In the brine shrimp lethality test, $\mathrm{LC}_{50}$ value of crude methanolic extract obtained from the best-fit line slope was $3.06 \mu \mathrm{g} / \mathrm{ml}$ which was significant comparing with control (vincristine sulphate). Above results suggested that the L. macrophylla can be regarded as a promising candidate for natural plant sources of antioxidants with high value.
\end{abstract}

Keywords: DPPH free radical, Total phenolic content, Brine shrimp lethality bioassay

\section{Introduction}

Oxidative stress is closely related with the pathogenesis of life-style diseases such as atherosclerosis, hypertension, diabetes mellitus, ischemic diseases, and malignancies. ${ }^{1}$ Oxygen consumption during metabolism generate different kinds of oxygen free radicals (ROS) namely superoxide radical, hydrogen peroxide, hydroxyl radical, singlet oxygen or nitrogenous substances (RNS) that can be hazardous for biological systems. $^{2}$ Majority of the ROS or RNS are removed or neutralized by the presence of enzymatic or nonenzymatic processes. ${ }^{3}$ Excess ROS can cause tissue damage which is thought to be the root cause of several other diseases such as arthritis, vasculitis, nephritis, emphysema, lupus erythematous, Alzheimer's diseases, Parkinson's diseases etc. ${ }^{4}$ Exogenous antioxidants such as medicinal herbs can be also used to reduce the oxidative damage exerted by different free radicals due to their availability and economic viability. ${ }^{5}$
Leeamacrophylla Roxburgh (Family: Leeaceae), a wild edible herb enriched with vitamins, is locally named as Dholsamudra, Dinda, Hatikana, Hastikarnapalash. $^{6}$ It is widely distributed in Dinajpur, Savar and Chittagong hill tracts of Bangladesh, China, Cambodia, India, Laos, Myanmar, Nepal and Thailand. Besides its edible values, the roots of the plant have been used traditionally in fracture and healing cut injury and to allay pain. It has astringent, styptic and antiseptic activity. ${ }^{7}$ Potential anti-inflammatory effect of L. macrophylla leaves has been reported in experimental rats. ${ }^{8}$ Previously, researchers have reported antinociceptive and antioxidant activities of different extracts of roots of L. macrophyllain swiss albino. ${ }^{9,10}$ So far, there are no scientific reports about antioxidant and cytotoxicity potentials of the leaves of L. macrophylla in the country.

In this paper, DPPH free radical scavenging assay, total phenolic content, total flavonoid content of the crude methanolic extract of the leaves of $L$. macrophylla were reported using spectroscopic 
technique. In addition, extract was evaluated its cytotoxic property using brine shrimp lethality bioassay.

\section{Material and Methods}

Chemicals and reagents: 1,1-Diphenyl-2picrylhydrazyl (DPPH), L-Ascorbic acid, Gallic acid, Quercetin, Folin-Ciocalteu reagent, Aluminium Chloride, Sodium carbonate, Sodium acetate, and Sodium chloride were purchased from Sigma Chemical Co. (St. Louis, MO, USA). Brine shrimp eggs were purchased from local marine market. All other reagents and chemicals were of $\mathrm{BDH}$ and $\mathrm{E}$ Merck of analytical grade.

Plant material: The fresh leaf of Leeamacrophylla was collected from local market of Natore district in the year of 2014 and was identified by $\mathrm{Mr}$. Mustafizur Rahaman, Assistant Professor, Department of Botany, Rajshahi University. A voucher specimen was deposited in the herbarium of Department of Botany for further references.

Extraction and isolation: The leaves were dried under shade, and powdered into coarse powder. Approximately $1 \mathrm{~kg}$ powdered leaf was taken and macerated for 7 days using $2 \mathrm{~L}$ of methanol with occasional stirring. The extract was filtered first with cotton then Whatman filter paper No. 1 to remove particulate matters and evaporated under reduced pressure in a rotary vacuum evaporator until all the solvent had been removed to give a semisolid residue (MELM) (85 gm, yield 8.5\%, $\mathrm{w} / \mathrm{w})$.

Determination of total phenolic content: Total phenolic content was determined with the FolinCiocalteu reagent according to a procedure described by Singleton and Rossi with slight modification. ${ }^{11,12}$ Briefly, $1 \mathrm{mg}$ of the crude extract was dissolved in $1 \mathrm{~mL}$ of $\mathrm{MeOH}$. $0.2 \mathrm{~mL}$ of the diluted sample was diluted with $1.8 \mathrm{~mL}$ of distilled water and reacted with $0.2 \mathrm{~mL}$ of Folin-Ciocalteu reagent for $5 \mathrm{~min}$, and then $2 \mathrm{~mL}$ saturated sodium carbonate solution (about $70 \mathrm{~g} / \mathrm{L}$ ) was added into the reaction mixture. $0.8 \mathrm{~mL}$ of distilled water was added to make the final volume of $5 \mathrm{~mL}$. The absorbance readings were taken at $750 \mathrm{~nm}$ after incubation at room temperature for $1.5 \mathrm{~h}$. Gallic acid was used as a reference standard, and the results were expressed as milligram gallic acid equivalent (mg GAE)/g dry weight of herbal material. Total phenolic content was calculated from the following calibration curve:

$$
\mathrm{Y}=0.006 \mathrm{X}+0.039, \quad \mathrm{R}^{2}=0.997
$$

Where $\mathrm{Y}$ is the absorbance of crude extract and $\mathrm{X}$ is the Gallic acid equivalent.

Determination of total flavonoid content: The total flavonoid content of crude extract was determined by the aluminum chloride colorimetric method. ${ }^{13}$ In brief, $1.5 \mathrm{~mL}$ of crude extract $(1 \mathrm{mg} / \mathrm{mL}$ Methanol) was mixed with $0.1 \mathrm{~mL}$ of $10 \% \mathrm{AlCl}_{3}$ and then 0.1 $\mathrm{mL}$ of $1 \mathrm{M} \mathrm{Na}$-acetate was added to the reaction mixture. The mixture was allowed to stand for 6 min. Then, $1 \mathrm{~mL}$ of $1 \mathrm{~mol} / \mathrm{L} \mathrm{NaOH}$ solution was added, and the final volume of the mixture was brought to $5 \mathrm{~mL}$ with double-distilled water. The mixture was allowed to stand for $15 \mathrm{~min}$, and absorbance was measured at $415 \mathrm{~nm}$. The total flavonoid content was calculated from a calibration curve, and the result was expressed as mg quercetin equivalent per $\mathrm{g}$ dry weight. Total flavonoid content was calculated from the following calibration curve:

$$
\mathrm{Y}=0.0067 \mathrm{X}+0.0132, \quad \mathrm{R}^{2}=0.973
$$

Where $\mathrm{Y}$ is the absorbance of crude extract and $\mathrm{X}$ is the quercetin equivalent.

1,1-Diphenyl-2-picryl-hydrazyl free radical scavenging assay: The free radical scavenging activity of crude methanolic extract of the plant was estimated by the method described by Feresin et al. using with the stable radical 1,1-diphenyl-2picrylhydrazyl (DPPH). ${ }^{14} 2.0 \mathrm{ml}$ of a methanol solution of the sample (extractives/control) at different concentration $(500-1 \mu \mathrm{g} / \mathrm{ml})$ were mixed with $3.0 \mathrm{ml}$ of a DPPH methanol solution (20 $\mu \mathrm{g} / \mathrm{ml})$. After $30 \mathrm{~min}$ reaction period at room temperature in dark place the absorbance was measured at $517 \mathrm{~nm}$ against methanol as blank by UV spectrophotometer. In hibition of free radical DPPH in percent (I \%) was calculated as follows:

$$
\mathrm{I} \%=\left(1-\mathrm{A}_{\text {sample }} / \mathrm{A}_{\text {blank }}\right) \times 100
$$

where, $A_{\text {blank }}$ is the absorbance of the control reaction (containing all reagents except the test material). The decrease in absorbance at $562 \mathrm{~nm}$ with the plant extracts and the reference compound indicates their abilities to quench superoxide radical in the reaction mixture. Lower absorbance of the reaction mixture indicated higher free radical scavenging activity.

Cytotoxic activity: Cytotoxic activity of the extract was determined by brine shrimp lethality bioassay technique. ${ }^{15}$ For the experiment, $4 \mathrm{mg}$ of extracts 
was dissolved in dimethyl sulfoxide (DMSO) and solutions of varying concentrations $(400,200,100$, $50,25,12.5,6.25,3.13,1.56,0.78 \mathrm{mg} / \mathrm{ml})$ were obtained by the serial dilution technique using simulated seawater. The solutions were then added to the pre-marked vials containing 10 live brine shrimp nauplii in $5 \mathrm{ml}$ simulated seawater. After 24 $\mathrm{h}$, the vials were inspected using a magnifying glass and the number of survived nauplii in each vial was counted. The mortality endpoint of this bioassay was defined as the absence of controlled forward motion during $30 \mathrm{~s}$ of observation. ${ }^{16}$ From this data, the percent of lethality of the brine shrimp nauplii for each concentration and control was calculated. An approximate linear correlation was observed when logarithm of concentration versus percentage of mortality was plotted on the graph paper and the values ofLC $\mathrm{C}_{50}$ were calculated using Microsoft Excel 2010.Vincristine sulphate was used as positive control.

Statistical analysis: Each test was replicated three times. Statistical analysis was carried out with GraphPad Prism 6 software (Graph Pad Software, Inc., USA), and results are expressed as means \pm standard deviation.

\section{Results}

Total phenolic and flavonoid content: The total phenolic content was carried out on the basis of absorbance value of crude methanolic extract, reacted with Folin-Ciocalteu reagent and compared with gallic acid equivalents. The total phenolic content of the methanolic leaf extract, calculated from the calibration curve $(\mathrm{R} 2=0.997)$, was found to be $65.75 \pm 1.20$ gallic acid equivalents/g. The way of determination of the total flavonoid content was also done on the basis of absorbance value of crude methanolic extract reacting with aluminum chloride. The total flavonoid content of the crude methanolic extract, compared with calibration curve ( $\mathrm{R} 2=0.993$ ), was $59.8 \pm 1.45$ quercetin equivalents $/ \mathrm{g}$ (table I). Phenolic compounds such as tannins, catechins and flavonoids have a number of hydroxyl groups which have redox properties, which allows them to act as antioxidants. As their free radical scavenging ability is facilitated by their hydroxyl groups, the total phenolic concentration could be used as a basis for rapid screening of antioxidant activity. Flavonoids, including flavones, flavanols, isoflavones, flavanonol and condensed tannins, are plant secondary metabolites, the antioxidant activity of which depends on the presence of free $\mathrm{OH}$ groups. Plant flavonoids have antioxidant activity in vitro and also act as antioxidants in vivo. ${ }^{17}$

Table I: Total phenolic and flavonoid contents of $\mathrm{MeOH}$ extract of L. Macrophylla

\begin{tabular}{|c|c|}
\hline Total phenolic content ${ }^{\mathrm{a}}$ & $65.75 \pm 1.20$ \\
\hline Total flavonoid content $b$ & $59.8 \pm 1.45$ \\
\hline
\end{tabular}

Antioxidant activity: The DPPH assay is based on the assumption that an antioxidant serves as a hydrogen donor and thus reduces(decolorizes) DPPH free radicals (the color turns from purple to yellow). This assay is very much common, easy and quick tool to evaluate antioxidant activity of putative antioxidants. Thus, the antioxidant potency of a compound is relative to the loss of DPPH free radicals (DPPH scavenging) that can be quantified through a decrease in the maximum absorption of DPPH at $570 \mathrm{~nm}$. A series of test tube were prepared to evaluate the inhibitory concentration of crude extract. The calibration curve was prepared using with ascorbic acid. Crude methanolic extract of $L$. Macrophylla showed $\mathrm{IC}_{50}$ values of $152.4 \mu \mathrm{g} / \mathrm{mL}$ whereas ascorbic acid showed $\mathrm{IC}_{50}$ values of 4.36 $\mu \mathrm{g} / \mathrm{mL}$.

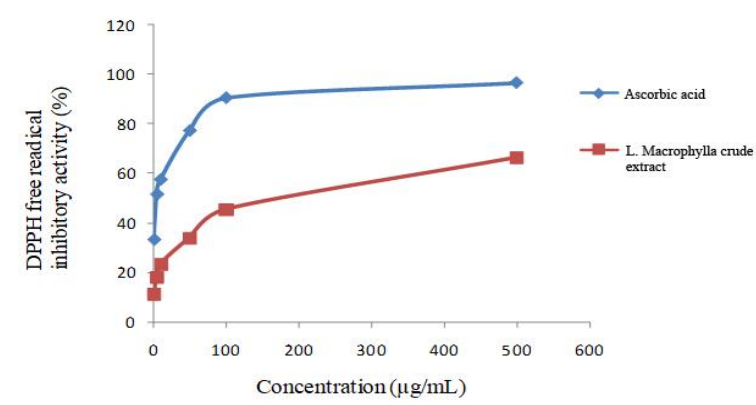

Figure 1: Dose-response curve of DPPH scavenging activity in the plant extract of $L$. Macrophylla.

Values represent reduction of DPPH radicals determined by measuring the absorption at $517 \mathrm{~nm}$. Each experiment was independently repeated as least three times from which standard deviations have been derived.

Cytotoxic activity: Brine shrimp lethality bioassay is a simple, high throughput cytotoxicity test of bioactive chemicals. It is based on the killing ability of test compounds on a simple zoological organismbrine shrimp (Artemiasalina). The brine shrimp lethality bioassay is widely used in the evaluation of toxicity of heavy metals, pesticides, medicines 
especially natural plant extracts and etc. It's a preliminary toxicity screen for further experiments on mammalian animal models. In brine shrimp lethality bioassay, $\%$ mortality increased gradually with the increase in concentration of the test samples (figure 2). The results of the brine shrimp lethality testing of methanolic extractives of $L$. Macrophylla after 24 hours of exposure to the samples and the positive control, vincristine sulphate (VS) (table II). The $\mathrm{LC}_{50}$ values were found to be 3.06 and $0.529 \mu \mathrm{g} / \mathrm{ml}$ for methanolic crude extract and VS respectively. In comparison to positive control (vincristine sulphate), the cytotoxicity exhibited by methanolic extract was promising. Values represent $\%$ mortality

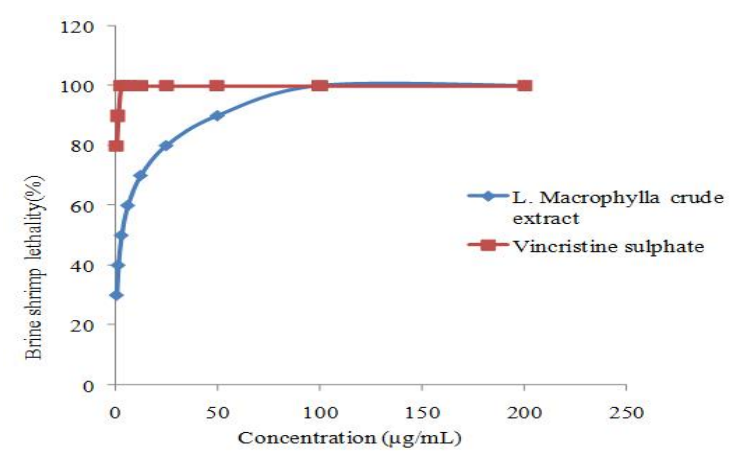

Figure 2: Dose-response curve of Brine shrimp lethality activity in the plant extract of L. macrophylla.

\section{Discussion}

Reactive oxygen species are generated normally in cells during metabolism. They can cause severe oxidative damage to proteins, lipids, enzymes and DNA by covalent binding and lipid peroxidation, with subsequent tissue injury. Researchers have shown their much interest on natural antioxidants because of their ability to scavenge free radicals without exerting toxic side effects. The presence of antioxidants such as phenolics, flavonoids, tannins and proanthocyanidins in plants may provide protection against a number of diseases; for example, ingestion of natural antioxidants has been inversely associated with morbidity and mortality from degenerative disorders. ${ }^{18}$ Medicinal plants are therefore being investigated for their antioxidant properties, and the demand for natural antioxidants and food preservatives is increasing. ${ }^{19}$

In the present study it was to be found that the methanolic leaves extract of L. Macrophylla contains substantial amount of phenolics and is being responsible for its marked antioxidant activity as assayed through various in vitro models. The plant contains substantial amount of flavonoids which was confirmed in total flavonoid content assay. Flavonoids are highly effective scavengers of most oxidizing molecules, including singlet oxygen, and various other free radicals implicated in several diseases. ${ }^{20}$ Flavonoids suppress reactive oxygen formation, chelate trace elements involved in free radical production, scavenge reactive species and up-regulate and protect antioxidant defenses. ${ }^{21}$ Similarly, phenolics conferring oxidative stress tolerance on plants. Crude extracts of fruits, herbs, vegetables, cereals and other plant materials rich in phenolics are increasingly being used in the food industry for their antioxidative properties and health benefits.

The free radical scavenging property was evaluated using neutralizing capacity towards DPPH free radical. The results showed that $L$. Macrophylla has significant free radical scavenging property. The result was correlated with the previous study where strong antioxidant capacity was reported from root part. ${ }^{10}$ The chemical composition and chemical structures of active extract components are important factors governing the efficacy of natural antioxidants. The antioxidant activity of an extract could not be explained on the basis of their phenolic content, which also needs their characterization.

The toxicity of the plants may originate from different contaminants or from plant chemical compounds that are part of the plant. Various assays are used for the research of potential toxicity of herbal extracts based on different biological models, such as in vivo assays on laboratory animals. In the present study the methanolic crude extract showed potent cytotoxic effects on brine shrimp. Previous phytochemical screening indicated the presence of alkaloids and flavones, which have been shown to possess cytotoxic activity, may be responsible in part for the antitumor effect on Ehrlich ascites carcinoma. $^{22}$ The plausible mechanism of cytotoxicity of L. Macrophylla against brine shrimp nauplii was due to poisonous effect on cell mitosis.

\section{Conclusion}

The plant possesses significant total phenolic and total flavonoid content which has potent antioxidant capacity. The different secondary metabolites are responsible for toxicity in human beings which can be exploited for anticancer activity in various cancer diseases. As the plant is a good source of secondary metabolites, the plant can be used for 
potential source of drug candidates which can be confirmed by modern research.

\section{Acknowledgement}

This project work was partially supported by the Grants on Advanced Research in Science, Ministry of Education, Bangladesh.

\section{References}

1. Maxwell SRJ. Prospects for the use of antioxidant therapies. Drugs. 1995; 49: 345-361.

2. Clark IA, Cowden WB, Hunt NH. Free radical induced pathology. Med Res Rev. 1985; 5:297-332.

3. Sies H. Biochemistry of oxidative stress. Angew ChemInt Ed. 1986; 25:1058-1071

4. Stefanis L, Burke RE, Greene LA. Apoptosis in neurodegenerative disorders. Curr Opin Neurol. 1997;10:299-305.

5. Chirag PJ, Tyagi S, Halligudi N, Yadav J, Pathak S, Singh SP, et al. Antioxidant activity of herbal plants: a recent review. J Drug Dis Ther. 2013;1:1-8.

6. Jadhao KD, Wadekar MP, Mahalkar MS. Remove from marked Records Comparative study of availability of vitamins from Leeamacrophylla Roxb. Biosci Biotechnol Res. 2009; 6:847-849.

7. Uddin SN. Traditional uses of ethnomedicinal plants of the Chittagong Hill Tracts. $1^{\text {st }}$ ed. Dhaka: Bangladesh National Herbarium; 2006.

8. Dewanjee S, Dua TK, Sahu R. Potential antiinflammatory effect of Leeamacrophylla Roxb. Leaves: A wild edible plant. Food Chem Toxicol. 2013; 59:514-520.

9. Mahmud ZA, Bachar SC, Qais N. Evaluation of antinociceptive activity and brine shrimp lethality bioassay of roots of LeeamacrophyllaRoxb. Int $\mathrm{J}$ Pharm Sci Res. 2011; 2:3230-3234.

10. Mahmud ZA, BacharSc, Hasan CM, Emran TB, Qais $\mathrm{N}$, and Uddin MMN. Phytochemical investigations and antioxidant potential of roots of Leeamacrophylla (Roxb.). BMC Res Notes. 2017; 10:1-9.
11. Singleton VL, Rossi JA. Colorimetry of total phenolics with phosphomolybdic- phosphotungstic acid reagents.Am J EnolVitic.1965; 16:144-158.

12. Kaur C, Kapoor HC. Anti-oxidant activity and total phenolic content of some Asian vegetables.Int J Food Sci Technol. 2002; 37:153-161.

13. Chang C, Yang M, Wen H, Chern J. Estimation of total flavonoid content in propolis by two complementary colorimetric methods. J Food Drug Anal. 2002; 10:178-182.

14. Feresin GE, Tapia A, Gutierrez RA, Delporte C, Backhouse EN, Schmeda HG. Free radical scavengers, anti-inflammatory and analgesic activity of Acaenamagellanica.J Pharm Pharmacol. 2002; 54:835-844.

15. Meyer BN, Ferrigni NR, Putnam JE, Jacobsen LB, Nichols DE, McLaughlin JL. Brine shrimp: A convenient general bioassay for active plant constituents. PlantaMedica. 1982; 45:31-34.

16. Apu AS, Muhit MA, Tareq SM, Pathan AH, Jamaluddin ATM, Ahmed M. Antimicrobial activity and brine shrimp lethality bioassay of the leaves extract of Dilleniaindica Linn. J Young Pharm. 2010; 2: $50-53$.

17. Shimoi K, Masuda S, Shen B, Furugori M, Kinze N. Radio-protective effects of antioxidative plant flavonoids in mice. Mutat Res Fund Mol. 1996; 350: 153-161.

18. Gulcin I. Antioxidant activity of food constituents: an overview. Arch Toxicol. 2012; 86:345-391.

19. Peschel W, Sanchez-Rabaneda F, Dieckmann W, Plescher A, Gartzia I, et al. An industrial approach in the search of naturalantioxidants from vegetable and fruits wastes. Food Chem. 2006; 97: 137-150.

20. Bravo L. Polyphenols: chemistry, dietary sources, metabolismand nutritional significance. Nutr Rev. 1998; 56:317-333.

21. Agati G, Azzarello E, Pollastri S, Tattini M. Flavonoids asantioxidants in plants: location and functional significance. Plant Sci. 2012; 96:67-76.

22. Brown JP. A review of genetic effects of occurring flavonoids, anthraquinones and related compounds. Mutation Res. 1980; 75:243-277.

*Correspondence: Firoj Ahmed, Department of Pharmaceutical Chemistry, University of Dhaka, Dhaka, Bangladesh; e-mail: firoj72@du.ac.bd 